\title{
Study materials lead to suspicion of an unnatural death-A case report
}

\section{B. Suresh Kumar Shetty, Jagadish Rao Padubidri*}

Department of Forensic Medicine, Kasturba Medical College (Affiliated to Manipal University), Mangalore, India;

*Corresponding Author: ppjrao@gmail.com

Received 27 December 2012; revised 31 January 2013; accepted 8 February 2013

Copyright (c) 2013 B. Suresh Kumar Shetty, Jagadish Rao Padubidri. This is an open access article distributed under the Creative Commons Attribution License, which permits unrestricted use, distribution, and reproduction in any medium, provided the original work is properly cited.

\begin{abstract}
The knowledge of human skeletal structure is a minimum requirement for all students who are practicing medicine. The skeletal makeup is used as a study material to acquire the knowledge of human anatomy. Skeletal study materials are obtained from the authorized markets by medical and paramedical students and teachers. The study materials are either kept at home or in their hostel rooms. After their basic medical study, they either keep safe these articles for their future reference or pass to their subordinate medical associates. The main problem arises if these skeletal structures are not disposed properly, after their study which may lead the Investigating officer to various medicolegal queries on recovery of these skeleton parts. Here is an interesting case, where the human bones were recovered by the municipality workers scattered in the garbage pit, which lead the investigating officer to register a case of unnatural death and begin with series of medicolegal query.
\end{abstract}

Keywords: Skeletal Remains; Conglomerate of Bones; Medico-Legal Autopsy

\section{INTRODUCTION}

Dissection of human body and study of human skeletal system forms an integral part of medical graduates. The studies have been practiced since centuries and are well depicted in our ancient surgical treasury written by Sushrutha. Human anatomy is also taught in relation to alternative medical systems such as Ayurveda, Homeopathy and Unani. A sound knowledge of human anatomy is needed to be well versed in surgery and medicine. It is said the great surgeons were good in their basic anatomical facts. This makes a rationale for training in anatomy, in the first year of Medical program of study. Skeletal arrangements meant for study purpose are obtained from the authorized markets by students and teachers. The study materials are either kept in home or in their hostel rooms. After concluding with their basic medical sciences, they either pass it to their subordinate medical students or hand it over to departmental museums in their medical schools. The main predicament arises if these skeletal structures are not disposed off properly after their study. This case report highlights and frames a need for making the guidelines for appropriate disposal of skeletal materials meant for learning purpose.

\section{CASE REPORT}

One fine day morning the municipality workers recovered a conglomerate of skeletal remains within a plastic bag in a garbage pit [Figure 1]. Suspecting a foul play, the same was informed to the concerned police station following which the investigating authority seized all the available skeletal bones and subjected it for medicolegal opinion to our department. The bag was opened and following bones were found.

Total number of bones is 36

Skull of an adult female, one with horizontally cleanly cut skull with vault and another without vault -2 in number.

Hip bones of both sides of an adult male -2 in number.

Hip bone of right side of an adult female- 1 in number

Right side Femur bone of male sex- 1 in number.

Left side Femur bone of female sex-1 in number

Male Sacrum-2 in number

Female Sacrum-1 in number.

Right side Tibia of male sex-1 in number. 


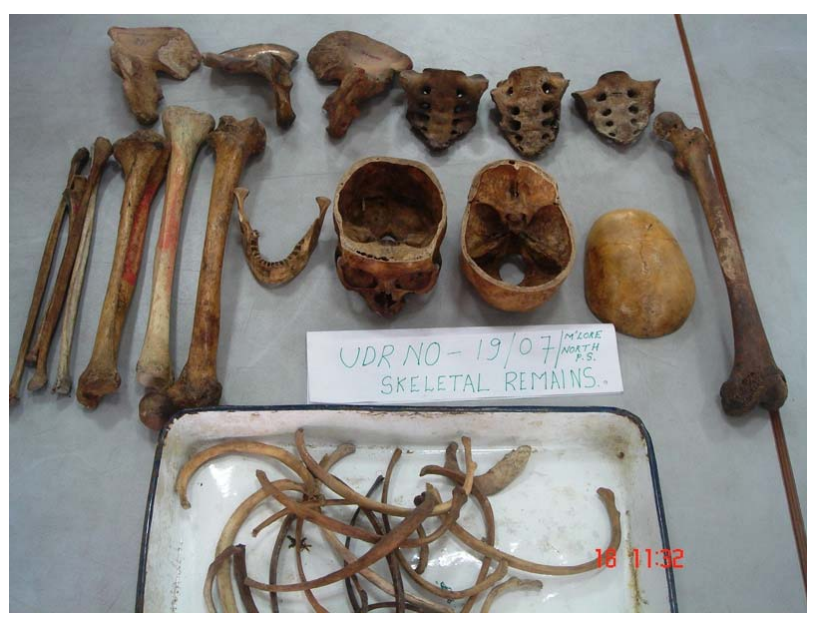

Figure 1. Human skeletal remains recovered from garbage bin, with cleanly cut vault of cranium.

Left side Tibia of female sex- 1 in number.

Left side Fibula, sex not determined -2 in number.

Right side Fibula, sex not determined -1 in number.

Left side Ulna , sex not determined-1 in number.

Ribs-20 in number, 11 were of right side and remaining 9 of left side.

Most of the bones were marked with red and green sketch pens, specially "Illiacus and G. Maximus" in the hip bone. A mark 'M.P' on the inner surface of hip bone., which clearly suggest that all the above bones were used by basic medical science students to study regarding the origin and insertion of the muscles during their anatomical tenure [Figures 2 and 3 ].

A detailed examination of skeletal remains revealed no evidence of ante mortem fractures. The parts of skeletal structure belonged to more than one individual. The disparity among sizes, non fusions of ossificational centers among bones indicative of different ages [Figure 4]. The bones did not show any evidence of trauma due to external trauma, so the specific question enquired by the investigating authority as death due to trauma could be ruled out.

It was opined that these bones subjected were dry, odourless, devoid of muscle tissues, varnished, manually had sketch pen markings, which suggests that these bones could have been used by medical and paramedical graduates for their academic purposes without having any suspicion.

\section{DISCUSSION AND CONCLUSIONS}

In the present case the retrieved skeletal remains had been used for study purposes and the sketch pen markings over it are the means in which the medical graduates learn and remember anatomical structures, which is a difficult subject for any medical student who have gone

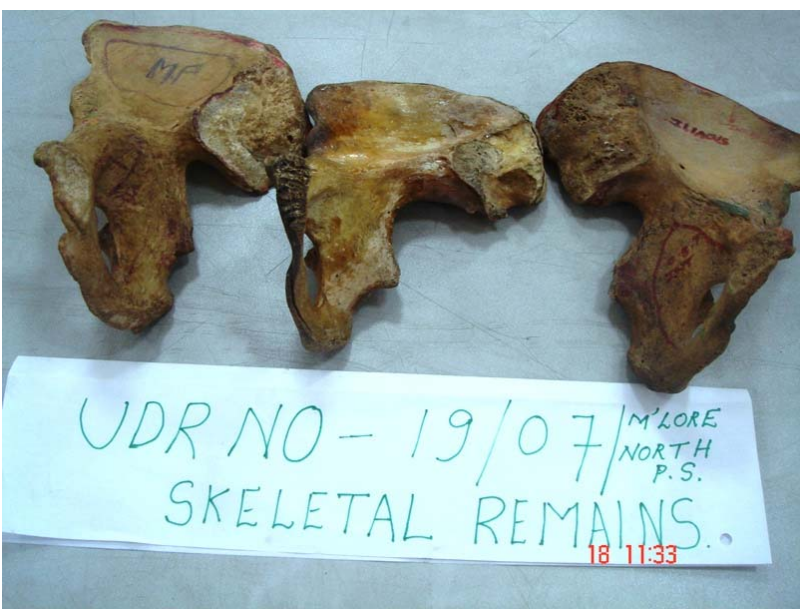

Figure 2. Cleanly varnished hip bones [3 in number] written as "MP" over the iliac bone.

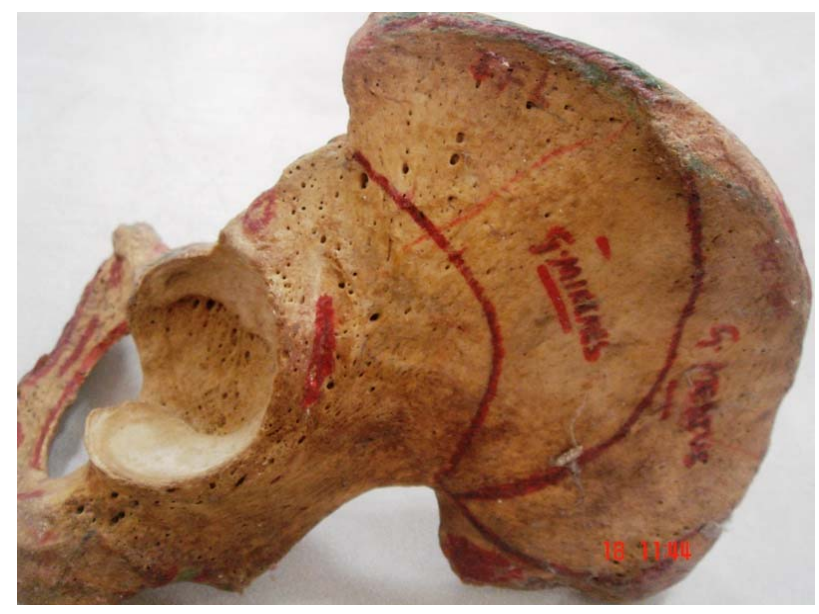

Figure 3. Cleanly macerated pelvic bone mentioning the names of the muscle attachments with red sketch pen.

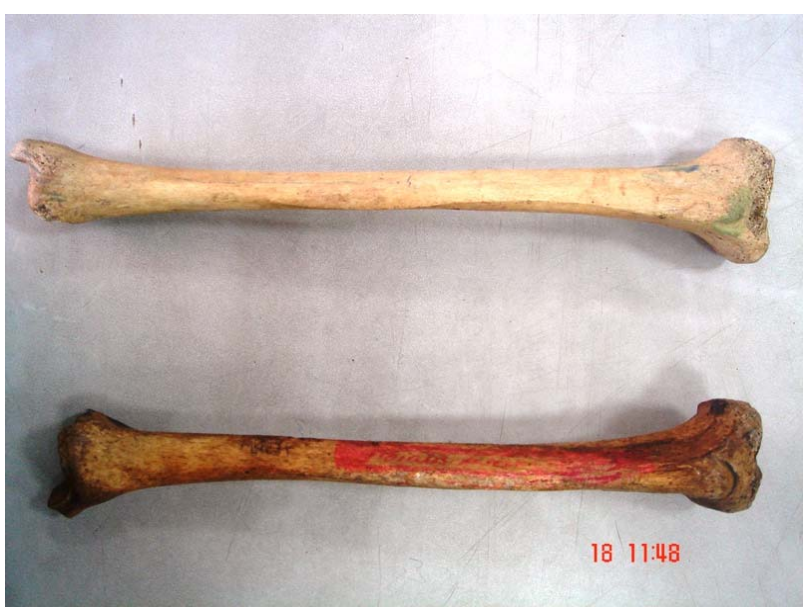

Figure 4. Cleanly varnished tibia of different length, with red sketch pen marks.

through anatomy. So the initiative mentioned here was to highlight how improper disposals of anatomical bones 
lead to unnecessary investigation in the event as described in the present case. Teachers of medical and paramedical schools should cultivate awareness among the medical graduates not to dispose the study materials like bones in public places or garbage's as highlighted in this particular case. There should be appropriate guidelines for the disposal of such study materials, especially the human skeletal bones.

However it must be remembered that throwing away of body parts is illegal as per Section 297 of the Indian Penal Code, 1860 and the person doing so can be charged for this offence [1].

According to this section:
Whoever acts as a depository for the remains of the dead or offers any indignity to any human corpse, shall be punished with imprisonment of either description for a term which may extend to one year or with fine or both [1].

Overall, any disrespect to dead body or body parts should be avoided.

\section{REFERENCES}

[1] Ratanlal and Dhirajlal's. (2012) The Indian penal code. 28th Edition, Reprint 2002, Wadhwa and Company Law Publishers, Indore. 